\title{
One-nucleon pickup reactions and compound-nuclear decays
}

\author{
J. E. Escher ${ }^{1, *}$, J. T. Burke ${ }^{1,}$, R. J. Casperson ${ }^{1,}$, R. O. Hughes ${ }^{1,}$, and N. D. Scielzo, \\ ${ }^{1}$ Lawrence Livermore National Laboratory, Livermore, CA 94550, USA
}

\begin{abstract}
One-nucleon transfer reactions, long used as a tool to study the structure of nuclei, are potentially valuable for determining reaction cross sections indirectly. This is significant, as many reactions of interest to astrophysics and other applications involve short-lived isotopes and cannot be measured directly. We describe a procedure for obtaining constraints for calculations of neutron capture cross sections using observables from experiments with transfer reactions. As a first step toward demonstrating the method, we outline the theory developments used to properly describe the production of the compound nucleus ${ }^{88} \mathrm{Y}^{*}$ via the one-nucleon pickup reaction ${ }^{89} \mathrm{Y}(\mathrm{p}, \mathrm{d})^{88} \mathrm{Y}^{*}$ and test the description with data from a recent experiment. We indicate how this development can be used to extract the unknown ${ }^{87} \mathrm{Y}(\mathrm{n}, \gamma)$ cross section from ${ }^{89} \mathrm{Y}(\mathrm{p}, \mathrm{d} \gamma)$ data. The example illustrates a more generally applicable method for determining unknown cross sections via a combination of theory and transfer (or inelastic scattering) experiments.
\end{abstract}

\section{Introduction}

One-nucleon pickup and stripping reactions have been used for decades in nuclear-structure studies. Most studies have focused on the low-energy $(\$ 2-3 \mathrm{MeV})$ properties of nuclei where single-particle properties are most clearly distinguished. Understanding excitations at higher energies, created by removing a nucleon from a deeply-bound orbital or by populating a resonance, is more challenging [1]. With the recent interest in using transfer reactions to indirectly infer cross sections for reactions involving very short-lived nuclei [2-6], it becomes relevant to revisit the issue.

The motivation for carrying out such transfer experiments stems from a need to determine unknown cross sections for nuclear astrophysics and other applications. Neutron capture cross sections, for instance, are crucial for understanding the production of the elements from iron to uranium that we observe today. It is well known that nucleosynthesis of heavy elements beyond ${ }^{56} \mathrm{Fe}$ takes place primarily by neutron capture on lighter seed nuclei in the s (slow neutron capture) and $\mathrm{r}$ (rapid neutron capture) processes, with other processes contributing to the abundances of some specific isotopes. Near stability, neutron captures on s-process branch points, unstable nuclei with a lifetime long enough to allow the s process to proceed by either neutron capture or $\beta$ decay, are of particular interest. The competition between capture and decay paths depends on environmental variables, such as neutron densities, temperatures, and pressure, as well as on nuclear properties. Measured s-process abundances, therefore, yield valuable insights into the detailed conditions of the astrophysical s process. Further away from stability, one finds isotopes for which the capture cross sections af-

*e-mail: escher1@llnl.gov fect the isotopic abundance patterns predicted by r-process models. While the $r$ process is known to take place in a high-temperature, high-flux environment, neither the exact path along the nuclear chart nor the astrophysical site where it takes place is known. Capture cross sections significantly affect the isotopic abundance patterns predicted by astrophysical models and need to be known to draw conclusions about stellar evolution and nucleosynthesis [7-9].

In this contribution, we will describe how transfer reactions can be employed to indirectly determine cross sections for reactions involving unstable (or highlyradioactive) isotopes. We will focus on understanding the ${ }^{89} \mathrm{Y}(\mathrm{p}, \mathrm{d})$ pickup reaction and indicate how this knowledge can be used to determine the cross section for neutron capture on the short-lived ${ }^{87} \mathrm{Y}$ isotope. In the next section, we will briefly review the formalism for compound neutron capture and show how a transfer reaction measurement, such as the (p,d) pickup reaction, can provide constraints for calculations of capture cross sections. In Section 3, we will outline the theory developments needed to describe compound-nucleus formation in the $(\mathrm{p}, \mathrm{d})$ reaction. We will show results from a recent experiment that serve as a test of the theory. Concluding remarks are offered in Section 4.

\section{Cross section calculations and constraints from indirect measurements}

Cross sections for many short-lived isotopes can be calculated, but suffer from large uncertainties due to poorlyconstrained inputs. Much effort has been devoted to develop models to calculate nuclear level densities and transmission coefficients for particles, photons, and fission, and 
to formulate parameter recommendations for compoundnuclear $(\mathrm{CN})$ reactions [10]. For low-energy neutron capture, nuclear level densities and $\gamma$-ray strength functions play a crucial role and need to be constrained. Auxiliary experiments provide important constraints for these models and their parameters. Most notably, neutron resonance measurements provide average level spacings and thus constraints for the level densities near the neutron separation energy [11]. Average radiative widths provide information on the product of the level density and the $\gamma$ ray strength function. However, for reactions involving unstable targets, the requisite neutron resonance spacings and average radiative capture widths are not available, and modeling the decay of the $\mathrm{CN}$ becomes the primary source of uncertainty in calculations of capture cross sections.

The CN capture reaction $n+A \rightarrow B^{*} \rightarrow \gamma+B$ is described in the statistical Hauser-Feshbach formalism $[12,13]$, which accounts for conservation of angular momentum and parity:

$$
\sigma_{n+A, \chi}\left(E_{n}\right)=\sum_{J, \pi} \sigma_{n+A}^{C N}\left(E_{e x}, J, \pi\right) G_{\chi}^{C N}\left(E_{e x}, J, \pi\right) .
$$

Here $\chi=\gamma+B$ denotes the exit channel, and the excitation energy $E_{e x}$ of $B^{*}$ is related to the neutron energy via $E_{n}=\frac{A+1}{A}\left(E_{e x}-S_{n}\right)$, where $S_{n}$ is the neutron separation energy of the nucleus $B$. Width fluctuation corrections have been omitted here, but have to be included in the calculations. The $\mathrm{CN}$ formation cross section, $\sigma_{n+A}^{C N}=$ $\sigma\left(n+A \rightarrow B^{*}\right)$, can be calculated using existing optical potentials. The theoretical decay probabilities $G_{\chi}^{C N}$ for the different decay channels $\chi$ are often quite uncertain, since they contain transmission coefficients, $T_{\chi l s}^{J}$, for all competing exit channels, the associated level densities, $\rho_{I}(U)$, in the various residual nuclei, as well as information on discrete levels:

$$
\begin{aligned}
& G_{\chi}^{C N}\left(E_{e x}, J, \pi\right)= \\
& \frac{\sum_{\ell s I} \int T_{\chi \ell s}^{J} \rho_{I}(U) d E_{\chi}}{\sum_{\chi^{\prime} \ell^{\prime} s^{\prime}}^{\prime} T_{\chi^{\prime} \ell^{\prime} s^{\prime}}^{J}+\sum_{\chi^{\prime} \ell^{\prime} s^{\prime} I^{\prime}} \int T_{\chi^{\prime} \ell^{\prime} s^{\prime}}^{J}\left(E_{\chi^{\prime}}\right) \rho_{I^{\prime}}\left(U^{\prime}\right) d E_{\chi^{\prime}}} .
\end{aligned}
$$

The quantities $\ell$ and $\ell^{\prime}$ are the relative orbital angular momenta in the exit channels and $\rho_{I}(U)$ is the density of levels of spin $I$ at energy $U$ in the residual nucleus. The denominator includes contributions from decays to discrete levels and to regions described by a level density. All sums over quantum numbers must respect parity conservation, although this is not explicitly expressed here.

In the absence of constraints on the level densities and $\gamma$-ray strength functions, the decay probabilities $G_{\chi}^{C N}\left(E_{e x}, J, \pi\right)$ become the primary source of uncertainty in calculations of capture cross sections. It is the purpose of indirect methods to provide constraints that reduce the uncertainties in the calculated cross sections.

In an approach that has become known as the 'surrogate method' [2], the compound nucleus $B^{*}$ is produced by an inelastic scattering or transfer reaction $d+D \rightarrow b$ $+B^{*}$, and the desired decay channel is observed in coincidence with the outgoing particle $b$ at angle $\theta_{b}$. The idea is schematically illustrated in Figure 1 for a one-nucleon pickup reaction, $\mathrm{p}+{ }^{A+2} Z \rightarrow \mathrm{d}+{ }^{A+1} Z^{*}$.

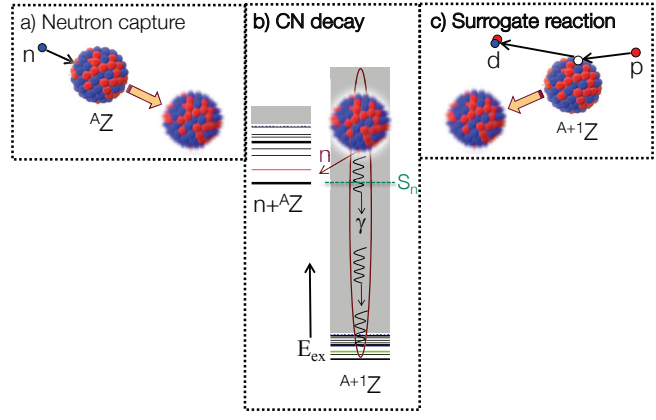

Figure 1. Surrogate approach, applied to a neutron-capture reaction. Neutron and target nucleus ${ }^{A} Z$ fuse to form the $\mathrm{CN}^{A+1} Z^{*}$ (panel a), which subsequently decays via a $\gamma$-cascade (panel b). Insufficient knowledge of nuclear properties results in a poor description of possible decay channels. In a surrogate experiment, the $\mathrm{CN}$ is produced by an alternative reaction, here a ${ }^{A+2} Z(\mathrm{p}, \mathrm{d})^{A+1} Z^{*}$ reaction (panel c). Observation of the subsequent decay of ${ }^{A+1} Z^{*}$ provides crucial constraints for the decay models.

The probability for forming $B^{*}={ }^{A+1} Z^{*}$ in the surrogate reaction (with specific values for $E_{e x}, J, \pi$ ) is $F_{\delta}^{C N}\left(E_{e x}, J, \pi, \theta_{b}\right)$, where $\delta$ refers to the surrogate reaction $d+D \rightarrow b+B^{*}$. The quantity

$$
P_{\delta \chi}\left(E_{e x}, \theta_{b}\right)=\sum_{J, \pi} F_{\delta}^{C N}\left(E_{e x}, J, \pi, \theta_{b}\right) G_{\chi}^{C N}\left(E_{e x}, J, \pi\right),
$$

which gives the probability that the $\mathrm{CN} B^{*}$ was formed with energy $E_{e x}$ and decayed into channel $\chi$, can be obtained experimentally by detecting a discrete $\gamma$-ray transition characteristic of the residual nucleus (or some other suitable observable).

The distribution $F_{\delta}^{C N}\left(E_{e x}, J, \pi, \theta_{b}\right)$, which may be very different from the $\mathrm{CN}$ spin-parity populations following the absorption of a neutron in the desired reaction, has to be determined theoretically, so that the branching ratios $G_{\chi}^{C N}\left(E_{e x}, J, \pi\right)$ can be extracted from the measurements. In practice, the decay of the $\mathrm{CN}$ is modeled using a HauserFeshbach-type decay model and the $G_{\chi}^{C N}\left(E_{e x}, J, \pi\right)$ are obtained by adjusting parameters in the model to reproduce the measured probabilities $P_{\delta \chi}\left(E_{e x}, \theta_{b}\right)$. Subsequently, the sought-after cross section can be obtained by combining the calculated cross section $\sigma_{\alpha}^{C N}\left(E_{e x}, J, \pi\right)$ for the formation of $B^{*}={ }^{A+1} Z^{*}$ (from $n+{ }^{A} Z$ ) with the extracted decay probabilities $G_{\chi}^{C N}\left(E_{e x}, J, \pi\right)$ for this state, see Eq. 1.

\section{Surrogate $(p, d)$ reactions: theory and experimental tests}

Generating accurate predictions of the surrogate spinparity $\left(J^{\pi}\right)$ distribution requires a model for the reaction mechanisms that are involved in the formation of the compound nucleus. Here we consider the example of ${ }^{89} \mathrm{Y}(\mathrm{p}, \mathrm{d})^{88} \mathrm{Y}^{*}$ which produces the $\mathrm{CN}^{88} \mathrm{Y}^{*}$. This $\mathrm{CN}$ is relevant to the ${ }^{87} \mathrm{Y}(\mathrm{n}, \gamma)$ reaction, to which we wish to apply 
the surrogate method. ${ }^{87} \mathrm{Y}$ has a half-life of 79.8 hours and current cross section calculations have to rely on regional systematics.

In the example considered here, the surrogate reaction produces ${ }^{88} \mathrm{Y}^{*}$ by removing neutrons from inner shells of the ${ }^{89} \mathrm{Y}$ nucleus: deep hole states are involved in the production of ${ }^{88} \mathrm{Y}^{*}$ near $S_{n}$. Their location and fragmentation as a function of $E_{e x}$ was obtained using the dispersive optical model approach of Mahaux and Sartor [14]. At the high excitation energies involved, one-step $(\mathrm{p}, \mathrm{d})$ pickup processes have to be complemented by contributions from two-step processes such as (p,p')(p',d) and (p,d')(d',d), in which the initial ${ }^{89} \mathrm{Y}$ or the final ${ }^{88} \mathrm{Y}$ are inelastically excited. The calculations were carried out using the coupledchannels code Fresco [15]. The proton-nucleus optical potential by Koning and Delaroche [16] was used to describe the entrance channel, and the deuteron-nucleus potential by Daehnick et al. [17] was employed for the exit channel. Due to the large number of states in the energy band populated, the different contributions can be assumed to add incoherently. Angle-integrated (p,d) cross sections can be calculated and compared to measured $(\mathrm{p}, \mathrm{d})$ cross sections.

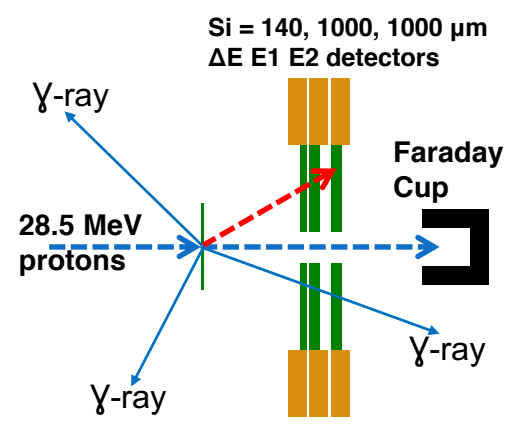

Figure 2. Experimental setup. A 28.5-MeV beam was incident on an ${ }^{89} \mathrm{Y}$ target. Outgoing deuterons were identified and their energy measured in a silicon telescope consisting of one thin and two thick detectors at angles covering $30-60^{\circ}$. Coincident $\gamma$ rays were detected in five HPGe clover detectors surrounding the target chamber.

The requisite experiment was carried out at the Texas A\&M University Cyclotron Institute, where the K150 Cyclotron was employed to provide a $28.5-\mathrm{MeV}$ proton beam. The setup is shown schematically in Figure 2. Particle- $\gamma$ coincidence data was collected using the STARLiTeR array, a combination of a silicon telescope array and five HPGe clover detectors [18]. The outgoing deuteron was detected at angles between $30^{\circ}$ and $60^{\circ}$ with $1^{\circ}-2^{\circ}$ angular resolution. From a reconstruction of the deuteron energies it was determined that the $\mathrm{CN}^{88} \mathrm{Y}$ was populated at excitation energies up to about $12 \mathrm{MeV}$. Contributions from carbon and oxygen contaminants were subtracted after measuring their characteristics using a carbon target within this particular setup. To provide information on the decay of the $\mathrm{CN}$, the experiment determined coincidences between the outgoing deuteron and $\gamma$-rays for transitions between low-lying states $\left(E_{n}<2 \mathrm{MeV}\right)$ in either ${ }^{88} \mathrm{Y}$ or ${ }^{87} \mathrm{Y}$, as a function of the excitation energy. More details about the experiment are given in Refs. [18-20].
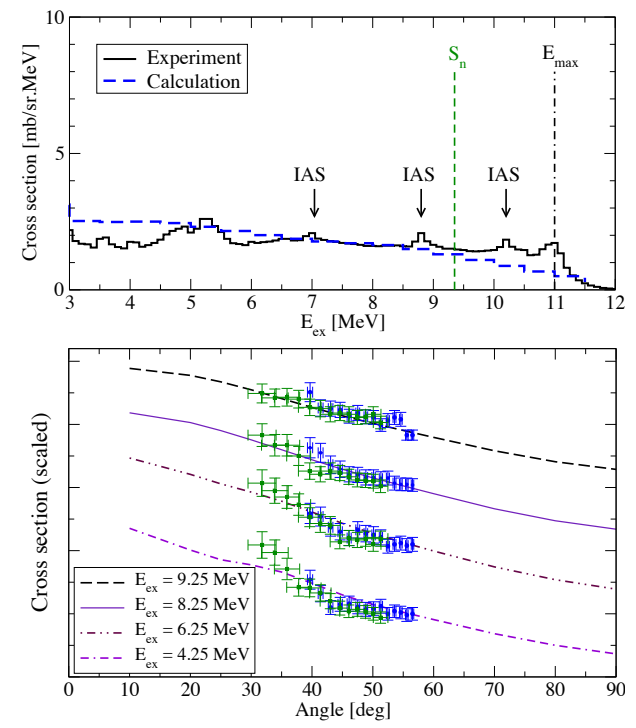

Figure 3. Comparison of the predicted ${ }^{89} Y(p, d){ }^{88} Y^{*}$ cross section to measured results. Top panel: The angle-integrated (p,d) cross section is shown as a function of excitation energy in ${ }^{88} \mathrm{Y}^{*}$. The calculation takes into account both one-step $(\mathrm{p}, \mathrm{d})$ and twostep $\left(\mathrm{p}, \mathrm{p}^{\prime}\right)\left(\mathrm{p}^{\prime}, \mathrm{d}\right)$ and $\left(\mathrm{p}, \mathrm{d}^{\prime}\right)\left(\mathrm{d}^{\prime}, \mathrm{d}\right)$ mechanisms. The cross section is integrated over the $30^{\circ}-60^{\circ}$ angular range covered by the experiment. The experiment also populated known isobaric ana$\log$ states ('IAS'); these are not explicitly included in the reaction calculation. $E_{\max }$ indicates the experimental upper limit and $S_{n}=9.35 \mathrm{MeV}$ is the neutron separation energy in ${ }^{88} \mathrm{Y}$. Bottom panel: Angular differential cross sections for the $(\mathrm{p}, \mathrm{d})$ reaction are shown for four excitation energies in the $\mathrm{CN}^{88} \mathrm{Y}$. The energies were selected to give a representative sample, without including the effects of the IAS. Data from two different detector segments are shown as blue and green circles with error bars. The cross sections were scaled for ease of comparison.

Preliminary results are shown in Figure 3, where we compare theoretical predictions to data from the $(\mathrm{p}, \mathrm{d})$ experiment. The dispersive optical model, which gives energy-averaged quantities, is not expected to reproduce the details of the rich nuclear structure present at low excitation energies $\left(E_{e x} \lesssim 2-3 \mathrm{MeV}\right)$. Hence we consider the results at higher energies $\left(E_{e x} \approx 3-10 \mathrm{MeV}\right)$. At those energies, two-step contributions are found to dominate the calculated (p,d) cross section, with one-step mechanisms contributing only about $20-25 \%$. The figure shows the sum of the two contributions as a dashed curve. Also, the experiment was seen to populate isobaric analog states, indicated with 'IAS' in the figure. The current reaction formalism does not predict cross sections for populating these states, 
but their effect on the spin distribution populated in the compound nucleus can be accounted for, since their spin and parity quantum numbers are known. Overall, the combination of the dispersive optical model with the two-step approach is found to give results that are in good agreement with the measurements, as can be seen in the top panel of Figure 3.

The characteristic angular shapes of one-nucleon transfers to low-lying discrete states are regularly used to extract useful information on the angular-momentum transfer in the reaction and, hence, on the possible spin and parity values of the final states. Here we focus on transfers to finite energy bins at higher excitation energies and see that the cross sections show little structure. Measurements for $E_{\text {ex }}=4.25 \mathrm{MeV}, 6.25 \mathrm{MeV}, 8.25 \mathrm{MeV}$, and 9.25 MeV (which are free of IAS contributions) show that the cross section is slightly decreasing, but relatively flat, in the $30^{\circ}$ $60^{\circ}$ range covered in the experiment, see bottom panel of Figure 3. The calculations reproduce this behavior well. Note that both the calculated and measured cross sections were scaled in order to allow for a clear angular comparison within the figure. The agreement of the absolute cross sections can be assessed by the comparison shown in the top panel.

The comparison of the predicted cross section to the data shows good agreement. This is an important step towards understanding the formation of a compound nucleus in a transfer reaction. For surrogate applications, the weights $F_{\delta}^{C N}\left(E_{e x}, J, \pi, \theta_{b}\right)$ of the final spins populated in the reaction have to be determined. This can be accomplished by calculating the relative contributions of the individual cross sections to the total (p,d) cross section.

The spin-parity distributions $F_{\delta}^{C N}\left(E_{e x}, J, \pi, \theta_{b}\right)$ obtained in this manner can then be combined with a model of the $\mathrm{CN}$ decay to reproduce the measured coincidence probabilities $P_{\delta \chi}\left(E_{e x}, \theta_{b}\right)$, as indicated in Equation 3. By varying the parameters in the decay models entering the expression for $G_{\chi}^{C N}\left(E_{e x}, J, \pi\right)$ and comparing the calculated $P_{\delta \chi}\left(E_{e x}, \theta_{b}\right)$ with the data, we can obtain constraints on these models and hence on the $G_{\chi}^{C N}\left(E_{e x}, J, \pi\right)$. This information is then used to calculate the desired neutron capture cross section for the short-lived ${ }^{87} \mathrm{Y}$ nucleus according to Equation 1. The results will be reported elsewhere [21].

\section{Conclusions}

One-nucleon transfer reactions are a valuable tool, not only for studying the structure of nuclei, but also for indirectly determining reaction cross sections. Many reactions of interest to astrophysics and other applications involve short-lived isotopes and cannot be measured directly. We discussed a procedure for obtaining constraints for calculations of neutron capture cross sections using observables from experiments with transfer reactions. Our specific example focused on the one-nucleon pickup reaction ${ }^{89} \mathrm{Y}(\mathrm{p}, \mathrm{d}){ }^{88} \mathrm{Y}^{*}$ which can be used to determine the neutron capture cross section for the short-lived ${ }^{87} \mathrm{Y}$ nucleus. We outlined the theory developments needed to properly describe the $\mathrm{CN}$ production via the transfer reaction and showed comparisons with experimental data.
This is a first, but critical step toward extracting the desired cross section from indirect $(\mathrm{p}, \mathrm{d} \gamma)$ data. The method outlined here properly takes into account the spins and parities populated in the surrogate reaction. Previous attempts to obtain (n, $\gamma$ ) cross sections from surrogate measurements invoked the approximation that either the decay probabilities $G_{\chi}^{C N}\left(E_{e x}, J, \pi\right)$ are independent of spin and parity or that the surrogate and desired reactions populate the $\mathrm{CN}$ in a similar manner. A number of theoretical [22-24] and experimental investigations [25-28] have demonstrated that these assumptions are not valid, with the region near ${ }^{90} \mathrm{Zr}$ being particularly sensitive to spin effects $[18,19,22,23,25]$.

The method outlined here for one-nucleon pickup reactions can also be employed with stripping reactions, such as $(\mathrm{d}, \mathrm{p})$, or with inelastic scattering. Several groups have revisited the formalism for inclusive $(\mathrm{d}, \mathrm{p})$ reactions [29-33], with the goal of describing transfers to both low-lying bound and highly-excited states above particle emission thresholds. All approaches have to be carefully tested and extended to apply to not only spherical but also deformed nuclei [34-36].

The longer-term goal is to develop a method that can be used for a wider range of reactions and a variety of projectile-target combinations. This makes it possible to reach a large number of isotopes, in particular when the technique can be combined with radioactive beams in inverse-kinematics experiments [37-39].

\section{Acknowledgments}

This contribution is dedicated to the memory of our friend and collaborator Cornelius Beausang, whose enthusiasm, optimism, and collegiality has been and will remain an inspiration. This work was performed under the auspices of the U.S. Department of Energy (DOE) by Lawrence Livermore National Laboratory under contract DE-AC52$07 N A 27344$.

\section{References}

[1] S. Gales, C. Stoyanov, A.I. Vdovin, Phys. Rep. 166, 125 (1988)

[2] J.E. Escher, J.T. Burke, F.S. Dietrich, N.D. Scielzo, I.J. Thompson, W. Younes, Rev. Mod. Phys. 84, 353 (2012)

[3] R. Raut, A.P. Tonchev, G. Rusev, W. Tornow, C. Iliadis, M. Lugaro, J. Buntain, S. Goriely, J.H. Kelley, R. Schwengner et al., Phys. Rev. Lett. 111, 112501 (2013)

[4] A. Spyrou, S.N. Liddick, A.C. Larsen, M. Guttormsen, K. Cooper, A.C. Dombos, D.J. Morrissey, F. Naqvi, G. Perdikakis, S.J. Quinn et al., Phys. Rev. Lett. 113, 232502 (2014)

[5] S.N. Liddick, A. Spyrou, B.P. Crider, F. Naqvi, A.C. Larsen, M. Guttormsen, M. Mumpower, R. Surman, G. Perdikakis, D.L. Bleuel et al., Phys. Rev. Lett. 116, 242502 (2016) 
[6] Escher, J. E., Tonchev, A. P., Burke, J. T., Bedrossian, P., Casperson, R. J., Cooper, N., Hughes, R. O., Humby, P., Ilieva, R. S., Ota, S. et al., EPJ Web of Conferences 122, 12001 (2016)

[7] A. Arcones, D.W. Bardayan, T.C. Beers, L.A. Bernstein, J.C. Blackmon, B. Messer, B.A. Brown, E.F. Brown, C.R. Brune, A.E. Champagne et al., Progress in Particle and Nuclear Physics 94, 1 (2017)

[8] Nuclear Science Advisory Committee, Reaching for the Horizon: The 2015 Long Range Plan for Nuclear Science (2015)

[9] M. Mumpower, R. Surman, G. McLaughlin, A. Aprahamian, Progress in Particle and Nuclear Physics 86, 86 (2015)

[10] R. Capote, M. Herman, P. Oblozinský, P. Young, S. Goriely, T. Belgya, A. Ignatyuk, A. Koning, S. Hilaire, V. Plujko et al., Nuclear Data Sheets 110, 3107 (2009)

[11] S.F. Mughabghab, Atlas of Neutron Resonances, Resonance Parameters and Thermal Cross Sections $Z=1-100,5$ th edn. (Elsevier, Amsterdam, 2006)

[12] W. Hauser, H. Feshbach, Phys. Rev. 87, 366 (1952)

[13] P. Fröbrich, R. Lipperheide, Theory of Nuclear Reactions (Clarendon Press, Oxford, 1996)

[14] C. Mahaux, R. Sartor, in Advances in nuclear physics (Springer US, 1991), Vol. 20, pp. 1-223

[15] I.J. Thompson, Computer Physics Reports 7, 167 (1988)

[16] A.J. Koning, J.P. Delaroche, Nucl. Phys. A713, 231 (2003)

[17] W.W. Daehnick, J.D. Childs, Z. Vrcelj, Phys. Rev. C 21, 2253 (1980)

[18] S. Ota, J.T. Burke, R.J. Casperson, J.E. Escher, R.O. Hughes, J.J. Ressler, N.D. Scielzo, I.J. Thompson, R.A.E. Austin, B. Abromeit et al., Phys. Rev. C 92, 054603 (2015)

[19] S. Ota, J. Burke, R. Casperson, J. Escher, R. Hughes, J. Ressler, N. Scielzo, I. Thompson, R. Austin, E. McCleskey et al., EPJ Web of Conferences 93, 02001 (2015)

[20] R.O. Hughes, J.T. Burke, R.J. Casperson, J.E. Escher, S. Ota, J.J. Ressler, N.D. Scielzo, R.A.E. Austin, B. Abromeit, N.J. Foley et al., Phys. Rev. C 93, 024315 (2016)

[21] J.E. Escher, J.T. Burke, R.O. Hughes et al., Constraining neutron capture cross sections for unstable nuclei with surrogate reaction data and theory, to be published (2018)
[22] C. Forssén, F. Dietrich, J. Escher, R. Hoffman, K. Kelley, Phys. Rev. C 75, 055807 (2007)

[23] J.E. Escher, F.S. Dietrich, Phys. Rev. C 81, 024612 (2010)

[24] S. Chiba, O. Iwamoto, Phys. Rev. C 81, 044604 (2010)

[25] N.D. Scielzo, J.E. Escher, J.M. Allmond, M.S. Basunia, C.W. Beausang, L.A. Bernstein, D.L. Bleuel, J.T. Burke, R.M. Clark, F.S. Dietrich et al., Phys. Rev. C 81, 034608 (2010)

[26] R. Hatarik, L.A. Bernstein, J.A. Cizewski, D.L. Bleuel, J.T. Burke, J.E. Escher, J. Gibelin, B.L. Goldblum, A.M. Hatarik, S.R. Lesher et al., Phys. Rev. C 81, 011602 (2010)

[27] G. Boutoux, B. Jurado, V. Méot, O. Roig, L. Mathieu, M. Aïche, G. Barreau, N. Capellan, I. Companis, S. Czajkowski et al., Physics Letters B 712, 319 (2012)

[28] Q. Ducasse, B. Jurado, M. Aïche, P. Marini, L. Mathieu, A. Görgen, M. Guttormsen, A.C. Larsen, T. Tornyi, J.N. Wilson et al., Phys. Rev. C 94, 024614 (2016)

[29] G. Potel, F.M. Nunes, I.J. Thompson, Phys. Rev. C 92, 034611 (2015)

[30] J. Lei, A.M. Moro, Phys. Rev. C 92, 044616 (2015)

[31] J. Lei, A.M. Moro, Phys. Rev. C 92, 061602 (2015)

[32] B.V. Carlson, R. Capote, M. Sin, Few-Body Systems 57, 307 (2016)

[33] G. Potel, G. Perdikakis, B.V. Carlson, M.C. Atkinson, W. Dickhoff, J.E. Escher, M.S. Hussein, J. Lei, W. Li, A.O. Macchiavelli et al., Eur. Phys. J. A 53, 178 (2017)

[34] B.L. Andersen, B.B. Back, J.M. Bang, Nucl. Phys. A 147, 33 (1970)

[35] B.B. Back, O. Hansen, H.C. Britt, J.D. Garrett, Phys. Rev. C 9, 1924 (1974)

[36] V. Gueorguiev, J. Escher, Bulg. J. Phys. 44, 489 (2017)

[37] J. Cizewski, R. Hatarik, K. Jones, S. Pain, J. Thomas, M. Johnson, D. Bardayan, J. Blackmon, M. Smith, R. Kozub, Nuclear Instruments and Methods in Physics Research Section B: Beam Interactions with Materials and Atoms 261, 938 (2007)

[38] A. Ratkiewicz, J. Cizewski, S. Pain, A. Adekola, J. Burke, R. Casperson, N. Fotiades, M. McCleskey, S. Burcher, C. Shand et al., EPJ Web of Conferences 93, 02012 (2015)

[39] J.A. Cizewski, A. Ratkiewicz, J.E. Escher, A. Lepailleur, S.D. Pain, G. Potel, EPJ Web Conf. 165, 01013 (2017) 